Rev Inv Vet Perú 2005; 16 (1):49-55

\title{
ENSAYO DE TRES PROGRAMAS DE VACUNACIÓN ANTICLOSTRIDIAL EN ALPACAS
}

\author{
Katherine Yaya L. ${ }^{1}$ y Raúl Rosadio A.,2
}

\section{AbSTRACT}

Enterotoxemia is the most important infectious disease in alpaca neonates. Its control depends on preventive handling measures and the application of immunoprofilaxis. The present study applied three programs of anticlostridial vaccination during three parturition seasons (years 2001, 2002, and 2003) in a cooperative farm in Puno. An anaculture with strains of Clostridium perfringens, predominantly of ovine origin, and a strain type A isolated from alpaca was used. The effect of the vaccine was evaluated by comparing rates of total and associated enterotoxemia mortality that occurred before the introduction of the vaccine (year 2000) in the farm. In the first program (year 2001), two doses of vaccine was administered to all pregnant mothers $(n=2,952)$ and one dose to all neonates. In the second program (year 2002), only newborns were vaccinated $(n=2,647)$ using one dose; and in the third program (year 2003) only pregnant mothers were vaccinated $(n=2,760)$ using one dose. The application of the vaccine reduced total neonatal mortality from 33.4 (year 2000) to 25.2 (year 2001), 23.7 (year 2002) and 9.4\% (year 2003). The vaccine equally reduced the mortality associated with enterotoxemia from 19.5 (year 2000) to $7.2,9.1$ and $1.0 \%$ in the same number of years. In conclusion, the anaculture (vaccine) was effective for the control of enterotoxemia in alpacas, independently of the type of application program; nevertheless, the reduction in mortality was greater in the program involving the vaccination of pregnant mothers.

Key words: enterotoxemia, alpaca, vaccine trials

\section{Resumen}

La enterotoxemia es la enfermedad infecciosa más importante que afecta las crías de las alpacas. El control está supeditado a la aplicación de medidas preventivas de manejo y a la aplicación de inmunoprofilaxis. En el presente trabajo se ensayaron tres programas de vacunación anticlostridial durante tres campañas de parición (2001, 2002 y 2003) en una unidad de producción de una empresa de propiedad social en Puno. Se empleó un anacultivo a base de cepas de Clostridium perfringens, predominantemente de origen ovino (cepas A, B, C y D) y de una cepa tipo A aislada de alpaca. El efecto de la vacuna se realizó comparando tasas de mortalidad total y mortalidad asociada a enterotoxemia ocurrida antes de la introducción de la vacuna (año 2000). En el primer programa (año 2001) se administró dos dosis de la vacuna a todas las madres gestantes $(n=2,952)$ y una dosis a todas las crías. En el segundo programa (año 2002) se vacunó con una dosis a todas las crías $(n=2,647)$, y en el tercer programa (año 2003) se vacunó con una dosis a

${ }^{1}$ CONOPA.E-mail: webmaster@conopa.org

${ }^{2}$ Laboratorio de Microbiología y Parasitología Veterinaria FMV-UNMSM

E-mail: rrosadioa@vet.unmsm.edu.pe; rrosadio@terra.com.pe 
todas las madres gestantes $(n=2,760)$. La aplicación de la vacuna redujo la mortalidad neonatal total de 33.4 (año 2000) a 25.2 (año 2001), 23.7 (año 2002) y a 9.4\% (año 2003). La vacuna, igualmente, redujo la mortalidad neonatal asociadas a enterotoxemia desde 19.5 (año 2000 ) hasta $7.2,9.1$ y $1.0 \%$ para los mismos años. Se concluye que el anacultivo (vacuna) fue efectivo para el control de enterotoxemia en alpacas, independientemente del tipo de programa aplicado. Sin embargo, la reducción fue más evidente en los programas que consideraron la vacunación de madres gestantes.

Palabras clave: enterotoxemia, alpaca, vacuna

\section{INTRODUCCrón}

La agroindustria de los camélidos sudamericanos es una actividad única e ideal para las condiciones de producción en las zonas altoandinas del Perú (Brenes et al., 2001). Sin embargo, el verdadero potencial econó mico de esta especie no se expresa a cabalidad, pues se encuentra limitada por diversos factores, entre ellos, el alto índice de mortalidad neonatal (Ramirez, 1987; Ramírez y Ellis, 1988).

La enterotoxemia es la enfermedad infecciosa responsable de las mayores pérdidas económicas que afectan a los productores alpaqueros (Ameghino y DeMartini, 1991; Moro, 1987; Ramírez, 1989, 1990). La enterotoxemia, denominada también diarrea bacilar, es una infección aguđa ocasionada por el Clostridium perfringens, que afecta principalmente a las crías entre la $2^{\mathrm{a}}$ y $3^{\text {a }}$ semana de edad (Ramirez et al., 1985). El cuadro patológico es consecuencia de un cuadro tóxico primario a nivel intestinal (yeyuno e ileon) que se deriva en un cuadro de toxemia generalizada a consecuencia de la acción de las toxinas del $C$. perfringens tipo $\mathrm{A}$ que ocasionan daños irreversibles en el endotelio vascular y el sistema nervioso. Generalmente, estas manifestaciones clínicas transcurren rápidamente y frnalizan con la muerte súbita del animal (Novoa y Florez, 1991).

La enfermedad se observa como brotes o epizootias, con mortalidades que pueden alcanzar el $50 \%$ del total de crías naci- das (Ramirez et al, 1985), constituyendo la principal causa de pérdidas neonatales en la producción de alpacas en el Perú (Novoa y Florez, 1991). La presentación de brotes epidémicos de la enfermedad, al parecer, es compleja, producto de las condiciones de manejo y posiblemente de la capacidad inmunológica de la cría; y está determinada por la presencia de los anticuerpos específicos transmitidos por la madre o de la propia respuesta inmune del neonato al agente causal (Ramírez y Elits, 1988; Ortíz, 1988).

Para prevenir la enterotoxemia se emplean medidas de higiene y manejo adecuadas, entre otras, la ingestión del calostro dentro de las primeras 12 horas de vida (Ameghino y DeMartini, 1991); sin embargo, muy pocas veces se implementan los calendarios de vacunaciones. El principal objetivo de la vacuna es proveer una inmunidad efectiva que se traduzca en mantener niveles adecuados de anticuerpos especificos y linfocitos memorias para una posterior activación rápida ante un nuevo contacto antigenico (Roitt, 1994).

Se dispone de antecedentes en el uso de productos biológicos comerciales para la prevención de enterotoxemia en alpacas en el pais (Ameghino y DeMartini, 1991), aunque existen experiencias preliminares que reducen las tasas de mortalidad neonatal con la aplicación de una vacuna anticlostridial (Moro, 1987). Por tanto, es necesario reiniciar estudios de campo que demuestren la habilidad de los productos biológicos en el control de la enfermedad clostridial en alpacas. Su análi- 
sis y difusión ayudará a superar las limitaciones inherentes a la situación del sector alpaquero del Perú.

\section{Materiales y Métodos}

\section{Localización}

El trabajo se realizó en la unidad de producción Huaripiña, de la Empresa de Propiedad Social Rural Alianza, en el distrito de Nuñoa (Melgar, Puno). La empresa ha estado dedicada a la crianza de alpacas por más de 20 años y mantiene una población promedio anual de 10,000 madres gestantes. La empresa nunca antes había aplicado vacunas contra la enterotoxemia.

\section{Diseño experimental}

La vacuna fue diseñada para proteger a una determinada población de animales buscando mantener inmune a un gran porcentaje de alpacas. Se compararon tres programas de vacunación aplicando la vacuna por 3 años consecutivos. En el programa 1 se vacunaron a todas las madres gestantes y a sus crías (año 2001); en el Programa 2 se vacunó a todos los neonatos (año 2002); y en el Programa 3 se vacunó a todas las madres gestantes (año 2003). Los detalles de los programas fueron los siguientes:

- Programa 1: Las madres $(\mathrm{n}=3,022)$ recibieron dos dosis de vacuna 30 y 15 días previos a la campaña de parición, y las crías una dosis a los 15 días de edad en promedio.

- Programa 2: Las crías $(n=2,500)$ recibiebieron una dosis de vacuna a los 15 días de edad en promedio.

- Programa 3: Las madres $(n=2,818)$ recibieron una dosis de vacuna 15 días previos a la campaña de parición.
Se utilizó como grupo control la parición correspondiente al año 2000 ( $\mathrm{n}=3,224$ crías), donde no se vacunó ni a las madres ni a las crías.

\section{Anacultivo}

La vacuna fue una suspensión del Clostridum perfringens y sus toxinas (anacultivo) formalinizadas y adsorbidas en hidróxido de aluminio. Las cepas componentes de la vacuna fueron $80 \%$ de origen ovino (C. perfringens tipos A, B, C y D) y $20 \%$ de una cepa tipo A aislada directamente de alpacas. La dosis de la vacuna (madres y crías) fue de $2 \mathrm{ml}$ administrada por vía subcutánea.

\section{Determinación de mortalidad neonatal}

La evaluación de la vacuna se hizo mediante el índice de mortalidad neonatal total (IMNT) y el índice de mortalidad neonatal por enterotoxemia (IMNE). Los índices de mortalidad fueron calculados en base a los registros de mortalidad de la empresa.

Se obtuvieron 20 muestras patológicas de animales que murieron con diagnóstico de enterotoxemia, a fin de monitorear la exactitud de los diagnósticos de campo. Estas muestras fueron remitidas para el aislamiento y tipificación bacterial al Laboratorio de Bacteriología de la Facultad de Medicina Veterinaria de la Universidad Nacional Mayor de San Marcos. Se realizaron cultivos bacteriológicos bajo condiciones anaeróbicas, tanto en medios líquidos (caldo carne) como en medios sólidos (agar sangre), así como la tipificación de las cepas aisladas por seroneutralización en ratones.

\section{Datos climáticos}

Los datos de humedad relativa media y precipitación total mensual fueron obtenidos de los registros metereológicos de la Oficina General de Estadística del SENAMHI. La información fue registrada desde 1998 al 2003 en la Estación de Chuquibambilla, distrito de Umachiri, en la provincia de Melgar, Puno. 
Cuadro 1. Indice de Mortalidad Neonatal Total (IMNT) e Índice de Mortalidad Neonatal por Enterotoxemia (IMNE) en un rebaño de alpacas sometidas a un programa de vacunación contra Clostridium perfringens

\begin{tabular}{lccccc}
\hline Programa de vacunación & Año & $\begin{array}{c}\text { Crias nacidas } \\
\text { vivas (n) }\end{array}$ & $\begin{array}{c}\text { IMNT } \\
(\%)\end{array}$ & $\begin{array}{c}\text { IMNE } \\
(\%)\end{array}$ & IC $^{1}$ \\
\hline 0: Control & 2000 & 3,224 & 37.4 & 19.5 & $18-21$ \\
1: Madre-Cría & 2001 & 2,952 & 25.2 & 7.2 & $6-8$ \\
2: Cría & 2002 & 2,647 & 23.7 & 9.1 & $8-10$ \\
3: Madre & 2003 & 2,760 & 9.4 & 1.0 & $0-1$ \\
\hline
\end{tabular}

${ }^{1}$ Intervalo de confianza del $95 \%$

\section{Resultados}

El Cuadro 1 muestra el número total de crías nacidas vivas, el indice de mortalidad neonatal total (IMNT) y el indice de mortalidad neonatal por enterotoxemia (TMNE) para cada campaña de parición. Se observa una disminución significativa de ambos indices entre el grupo control y los tres programas de vacunación $(p<0.05)$.

No se encontró diferencia estadística entre los programas de vacunación, aunque hubo una tendencia a mejores resultados con el programa de vacunación a las madres (año 2003). El análisis de varianza de una sola vía, por otro lado, determinó que los factores de precipitación pluvial y humedad relativa fueron estadísticamente homogéneos durante las cuatro campañas de parición.

En el laboratorio se confirmó que los diagnósticos de campo de enterotoxecnia fueron correctos. Se aisló el Clostridium perfringens en forma abundante, puro y con las características propias de Clostridium, verificadas por la tinción de Gram y pruebas químicas. Estas cepas fueron patogénicas para ratones, $y$ a través de seroneutralización se les identificó como cepas del tipo A.

\section{Discuisión}

Para evitar infecciones de impacto en etapas tempranas del neonato, como el caso de enterotoxemia en alpacas, se recomienda por lo general la vacunación de madres gestantes (Outteridge, 1985), de allí que dos de los programas ensayados involucraron la vacunación de madres gestantes. Sin embargo, en el campo los ganaderos son renuentes al manipuleo de animales preñados por el temor a problemas de estrés asociados con abortos. Por esta razón, se incluyó el programa 3 que implicaba vacunar únicamente a las crías.

Los resultados de este trabajo evidencian que los tres programas de vacunación ensayados redujeron dramáticamente los índices de mortalidad general, así como el índice de mortalidad neonatal debido a enterotoxemia. Los programas que involucran vacunaciones a madres gestantes ( 1 y 3 ) fueron aparentemente los más efectivos en la protección contra la enfermedad. Estos resultados permiten inferir que, independientemente del tipo de programa de vacunación aplicado, la vacuna anticlostridial es efectiva para el control de enterotoxemia en alpacas neonatas. Asi mismo, refuerzan la idea de que la enfermedad afecta a las crías, principal- 
mente entre la segunda y tercera semana de edad (Ramírez et al., 1985), periodo crítico que debe ser protegido por los anticuerpos maternales de la madre vacunada antes del parto (Bravo et al., 1997).

La vacunación exclusiva de crías también reduce sustancialmente los índices de mortalidad neonatal. De aceptarse este resultado, la vacunación a crías tendría una ventaja aplicativa en el campo, pues se evitaría la manipulación de las madres gestantes. Sin embargo, es posible que la aparente protección contra la enfermedad obtenida al vacunar a las crías sea debido a la presencia de anticuerpos maternales residuales provenientes de madres vacunadas el año anterior (Outteridge, 1985); de allí que habría que realizar estudios adicionales para comprobar las bondades de este programa de vacunación.

Por otro lado, la reducción de mortalidades por enterotoxemia después de la introducción inicial de la vacuna en el 2001 podría deberse a la denominada "inmunidad masal". Las coberturas de vacunaciones en los 3 años que duró el ensayo en la unidad experimental superó el 95\% de las hembras gestantes. El concepto de "protección masal" se aplica fundamentalmente para la prevención de enfermedades altamente infecciosas (Van Oirschot, 2001), pero igualmente, puede tener cierta validez en las infecciones asociadas a altas morbilidades y mortalidades como el caso de las enfermedades clostridiales en alpacas neonatas (Ameghino y DeMartini, 1991; Moro, 1987).

Este concepto, podría igualmente explicar la denominada ciclicidad de los brotes enterotoxigénicos en las explotaciones alpaqueras, la que se halla asociada a mayores precipitaciones pluviales (Ramírez, 1987). La ciclicidad, igualmente puede explicarse por la presencia de protección por anticuerpos en el hato después de brotes naturales de la enfermedad. Los animales sobrevivientes de brotes epizoóticos mantendrían niveles de anticuerpos en cantidades suficientes para proteger al rebaño por un determinado nú- mero de años. La disminución progresiva de la cantidad y calidad de anticuerpos en el hato y en los individuos del hato, incrementarían la susceptibilidad a nuevos desafíos de campo. En este contexto, mantener programas de vacunación con coberturas mayores al $80 \%$ ayudaría a reducir los brotes epizoóticos de la enfermedad en estos animales.

Así mismo, llama la atención que un anacultivo conteniendo Clostridium perfringens tipos A, B, C y D con sus respectivas exotoxinas $\propto, \beta$ y $\varepsilon$ haya protegido adecuadamente contra infecciones a Clostridium perfringens tipo A. La etiopatogenia de esta enfermedad, al parecer, depende de la habilidad de producir la enterotoxina (CPE), cuya liberación ocurre solamente en la fase de esporulación (Ramírez, 1987). La preparación del anacultivo descarta la presencia de la enterotoxina en la vacuna bajo prueba. $\mathrm{Si}$ los anacultivos protegen adecuadamente frente a infecciones clostridiales como ocurrió en el presente estudio, induciría a pensar que las exotoxinas $(\propto, \beta$ y $\varepsilon$ ) deberían, además de la enterotoxina, jugar roles patogénicos importantes en el desencadenamiento de la enfermedad en los neonatos.

La habilidad protectiva del anacultivo en la enterotoxemia de las alpacas se ha descrito desde los estudios iniciales de la enfermedad en el Perú (Moro, 1987) y más recientemente en Chile (Prehn et al., 1999). Este tipo de vacuna es muy usada en programas de control de la enfermedad, en países que han comenzado con crianza intensivas de alpacas en el resto del mundo (Fowler, 1998). Los anacultivos son excelentes biológicos, ampliamente usados en la protección contra el complejo enterotoxigénico en explotaciones ovejeras de muchos países, incluyendo el Perú (Rosadio y Ameghino, 1999).

La disminución de los índices de mortalidad a consecuencia de introducción de programas de vacunación se observó desde el primer año y comenzaron a decrecer dramáticamente en los años subsiguientes. La introducción de la vacuna redujo la mortalidad 
neonatal general en un $74.9 \%$ y la mortalidad debido a enterotoxemia en un $95 \%$ con respecto al reportado en la campaña de parición previo a la introducción de la vacuna. De esto se deduce que el mantenimiento de un nivel óptimo de anticuerpos transmisibles a la cría a través del calostro es más que suficiente para disminuir el número de muertes por causa de la enfermedad. Por otro lado, la vacuna, contrario al pensar de los ganaderos, no es abortigénica pues los porcentajes de abortos durante las cuatro campañas de parición fueron estables y semejantes en los años estudiados.

El presente estudio evidencia que un anacultivo polivalente reduce dramáticamente los índices de mortalidad neonatal general y la mortalidad neonatal debido a enterotoxemia en alpacas neonatas. La vacuna controla la enfermedad cuando se administra a madres $\mathrm{y}$ crías, sólo a madres y aún solamente en crías.

\section{Agradecimientos}

Los autores agradecen a Laboratorios Provet S.A.C. por la preparación de la vacuna; a los M.V. Walter Pinto, Teodoro Quispe y José Luis Gómez de la E.P.S. Rural Alianza por permitirnos realizar el trabajo de campo; a la Biol. Dora Huamán, Biol. Sonia Calle y M.V. María Cerón del Laboratorio de Bacteriología de la Facultad de Medicina Veterinaria-UNMSM y al Crnel. FAP. Rafael Campos, de la Oficina de Estadística e Informática del SENAMHI, por su importante contribución en el desarrollo del trabajo de investigación.

\section{LITERATURA CTTADA}

1. Ameghino, E.; J. DeMartini. 1991. Mortalidad en crías de alpacas. Boletín de Divulgación del Instituto Veterinario de Investigaciones Tropicales y de Altura (IVITA) UNMSM. Lima, Perú. p 71-80.

2. Bravo, P.; J. Garnica; M. Fowler. 1997. Immunoglobulin $G$ concentrations in periparturient llamas, alpacas and their crias. Small Ruminant Res. 26: 145-149.

3. Brenes, $E$.; K. Madrigal; $\boldsymbol{E}$ Pérez; K. Valladares. 2001. El cluster de los camélidos en Perú: Diagnóstico competitivo y recomendaciones estratégicas. Disponible en: http://www.caf.com/ attach/4/default/CamelidosPeru.pdf

4. Fowler, M. 1998. Medicine and surgery of South American Camelids: llama, alpaca, vicuña, guanaco. Iowa State University Press, AMES. $2^{\text {nd }}$ ed. p 166169.

5. Moro, M. 1987. Enfermedades infecciosas de las alpacas. Revista de Camélidos Sudamericanos 4: 7-31.

6. Novoa, C.; A. Florez. 1991. Producción de rumiantes menores: alpacas. Ed. RERUMEN. Lima. 311 p.

7. Ortíz, S. 1988. Evaluación de algunos métodos de control de la mortalidad en crías de alpaca (Lama pacos) en explotaciones familiares. Tesis de Bachiller. Facultad de Medicina Veterinaria, Univ. Nacional Mayor de San Marcos. Lima. $58 \mathrm{p}$.

8. Outteridge, P. 1985. Veterinary immunology. Academic Press. London, U.K. 280 p.

9. Prehn, R.; S. Saez; M. Arriagada. 1999. Estudios microbiológicos y clínicos de enterotoxemia por Clostridium perfringens en camélidos chilenos. II Congreso Mundial de Camélidos Sudamericanos. Cuzco, Perú.

10. Ramírez, A. 1987. Alpaca Clostridium perfringens type A enterotoxemia: purification and assays of the enterotoxin. Colorado State University. 201 p.

11. Ramírez, A. 1989. Enfermedades infecciosas en camélidos sudamericanos. XII Reunión Científica Anual de la Asociación Peruana de Producción Animal. p 85-105.

12. Ramirez, A. 1990. Avances sobre investigación en Salud Animal - Camélidos Sudamericanos. Boletín de Divulgación del Instituto Veterinario de Investigación Tropicales y de Altura (IVITA) UNMSM. Lima-Perú. No 23: 11-19. 
13. Ramírez, A.; D. Huamán; R. Ellis. 1985. Enterotoxemia de la alpaca. Reporte Técnico $\mathrm{N}^{\circ} 63$. INIPA y SR-CRSPLima. $56 \mathrm{p}$.

14. Ramírez A.; R. Ellis. 1988. Nuevos conceptos sobre la enterotoxemia y la colibacilosis en alpacas. Revista de Camélidos Sudamericanos 6: 9 -14.

15. Roitt, I. 1994. Essential immunology. $8^{\text {th }}$ ed. Blackwell Scientific Publications. $448 \mathrm{p}$.

16. Rosadio, R.; E. Ameghino. 1999. Enfermedades de los ovinos en el Perú. Publicación Técnica, FMV, UNMSM. Vol. 40.

17. Van Oirschot, J. 2001. Present and future of veterinary viral vaccinology: A review. Vet. Quarterly 23: 100-108. 